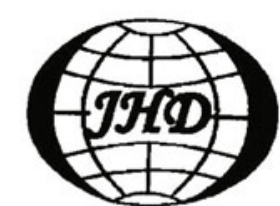

www.sciencedirect.com/ science/journal/10016058

\title{
Call For Paper \\ The 9th International Workshop on Ship and Marine Hydrodynamics (IWSH'2015, 26- 28 August 2015, Glasgow, UK)
}

In 1999, the first International Workshop on Ship Hydrodynamics was successfully held in Wuhan, China. Then it was held in Wuhan, Shanghai, Zhenjiang, Harbin, Seoul in subsequent every two years. These Conferences had drawn a variety of international delegates including scientists, engineers and researchers with a common interest in ship hydrodynamics. They provided a forum to promote scientific advancement, technological progress, information exchange, and cooperation among engineers and researchers in ship and ocean engineering and other related fields.

The coming IWSH'2015 conference will change its full name to International Workshop on Ship and Marine Hydrodynamics. It will be hosted and organized by University of Strathclyde, UK, and co-organized by editorial board of Journal of Hydrodynamics, as well as sponsored by Lloyd's Register, UK. The IWSH'2015 will be a platform for engineers and researchers to keep abreast of the current scientific and technological advancements in hydrodynamics of marine, ship and ocean engineering and other related fields, including theoretical, numerical and experimental aspects. Excellent papers submitted to IWSH'2015 will be selected to publish in Ocean Engineering and Journal of Hydrodynamics.

The IWSH'2015 Conference welcomes papers in aspects of hydrodynamics of ship and ocean engineering and other related fields. Specific themes include, but are not limited to:

-Resistance, propulsion, seakeeping, maneuverability
- Cavitation and cavitation flows

- Linear and nonlinear waves and current

-Slamming, sloshing, green water, run-up, impact

- Computational fluid dynamics, numerical wave tank and virtual displaying system

-Hull form optimization based on CAD/CFD

-Fluid-structure interaction and hydro-elasticity

-Environmental loads and underwater noise

-Hydrodynamic performance of high speed vessels

-Hydrodynamic performance of offshore platforms, pipelines, risers and mooring systems

-Hydrodynamics for renewable marine energy and ocean resources

-Experimental techniques for towing tank, wave flume and water basin

- Other aspects of hydrodynamics in ship and ocean engineering

More updated information of the IWSH'2015 conference can be found at http://www.iwsh2015.org

Conference abstract and full paper can be submitted via email before March 10, 2015 to:

Email: qing.xiao@strath.ac.uk, e.wang@strath.ac.uk

Dr. Qing Xiao

IWSH'2015 Secretary General, c/o

Department of Naval Architecture Ocean and Marine Engineering,

Strathclyde University, UK 